\section{$\underset{\substack{\text { hommes } \\ \text { \& migrations }}}{ }$}

\section{Hommes \& migrations}

Revue française de référence sur les dynamiques

migratoires

1326 | 2019

Londres et ses migrations

\title{
Londres : un refuge pour les exilés du Bahreïn
}

\section{Claire Beaugrand}

\section{QpenEdition \\ Journals}

\section{Édition électronique}

URL : https://journals.openedition.org/hommesmigrations/9689

DOI : 10.4000/hommesmigrations.9689

ISSN : 2262-3353

\section{Éditeur}

Musée national de l'histoire de l'immigration

\section{Édition imprimée}

Date de publication : 1 juillet 2019

Pagination : 69-75

ISBN : 978-2-919040-46-9

ISSN : $1142-852 X$

\section{Référence électronique}

Claire Beaugrand, «Londres : un refuge pour les exilés du Bahreïn », Hommes \& migrations [En ligne], 1326 | 2019, mis en ligne le 01 janvier 2022, consulté le 16 janvier 2022. URL : http://

journals.openedition.org/hommesmigrations/9689; DOI : https://doi.org/10.4000/

hommesmigrations.9689 


\section{Londres: un refuge pour les exilés du Bahreïn}

Claire Beaugrand, Lecturer, Institute of Arab and Islamic Studies, University of Exeter.

Les relations entre le Bahreïn, centre névralgique de l'Empire britannique dans la région du Golfe, et le Royaume-Uni sont marquées par leur héritage colonial. Londres a longtemps interféré dans les affaires politiques de l'île et introduit des pratiques de bannissement et d'exil forcé des individus s'opposant à ses intérêts. Ironiquement, la capitale britannique est devenue, un demi-siècle plus tard, un refuge privilégié pour les exilés bahreïniens en proie à la répression politique dans leur pays d'origine. Les générations de leaders politiques bahreïniens s'y succèdent ainsi, donnant lieu, paradoxalement, à la structuration d'une opposition en exil dans le pays comptant parmi les plus solides alliés de la dynastie en place.

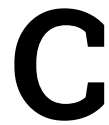

ontrairement aux autres monarchies du Golfe, plus enclines à la cooptation ou à la négociation avec les opposants au régime, le Bahreïn a une longue tradition d'exil forcé de son opposition politique. Cette singularité s'explique pour une part par l'héritage colonial britannique. En effet, l'Empire britannique avait fait de ce petit archipel sa principale base dans le Golfe: dès 1900, les Britanniques y postèrent un agent politique avant que Manama ne devienne, en 1947 lors de l'indépendance de l'Inde, le siège de l'administration coloniale pour l'ensemble de la région du Golfe, en remplacement de Bushehr, en Iran. Pour les Britanniques, il était primordial d'éviter toute forme de contestation sur ce petit archipel, de sorte que les leaders des mouvements de protestation furent condamnés à l'exil et déportés vers d'autres territoires de l'empire, qu'il s'agisse de Bombay, lors d'un soulèvement au début des années 1920, ou de l'île de Sainte-Hélène, après les événements de 1956, lors des protestations anti-impérialistes dans le sillage de la crise de Suez. Après l'accession du Bahreïn à l'indépendance en 1971, cette pratique s'est poursuivie alors que la monarchie voyait l'établissement d'un système politique pluraliste et démocratique, incluant des mouvements d'opposition (parfois ouvertement révolutionnaires), comme une menace pour sa survie.

Le Bahreïn n'est pas le seul pays à avoir recouru à cette pratique mais, contrairement à d'autres régions du monde, comme l'Amérique latine, où le rôle de l'exil politique a été davantage étudié et analysé, ce phénomène reste assez méconnu dans le monde arabe, où les récits d'exil convoquent immédiatement l'image de l'exode palestinien et de sa diaspora en Occident. Londres, en tant que capitale de l'ancien empire, a constitué un refuge pour les 
exilés politiques du Moyen-Orient dans divers contextes - et notamment dans le cas des chutes de régime mis en place par la puissance coloniale ou mandataire dans la région. Un des cas les plus marquants a été, par exemple, la chute sanglante, en 1958, de la monarchie hachémite, issue des shérifs de La Mecque, installée en Irak en 1921 par les Britanniques qui en assuraient le mandat. C'est en effet Londres qui sera la destination d'exil des partisans du système monarchique. Ils avaient été eux-mêmes précédés dans cet exil londonien par une partie de la communauté juive irakienne, dont ses plus grandes familles marchandes refusaient de migrer vers le nouvel État hébreu proclamé à la suite du départ des Britanniques de la Palestine mandataire. Plus tard, Londres accueillera aussi divers membres de l'opposition aux régimes autoritaires arabes, parfois au terme de trajectoires d'exil sinueuses, comme ce fut le cas du cheikh Rachid Ghannouchi du mouvement islamiste tunisien Ennahda, dont la demande d'asile fut refusée par la France.

Dans ce contexte historique large, on peut noter l'évolution de la composition sociologique des exilés issus de la péninsule arabique: à l'origine, l'exil était un phénomène élitaire lorsque, dans un contexte d'émergence étatique, les luttes de pouvoir et la contestation politique étaient limitées à un petit groupe de dynasties rivales ou déchues du pouvoir (comme les dynasties des sultanats du Yémen du Sud). Le phénomène a pris de l'ampleur lors de l'émergence - et de la répression de mobilisations populaires en faveur d'une participation plus inclusive. Par exemple, en Arabie saoudite, la rébellion des chiites dans la province orientale dans les années 1980 a ouvert de nouvelles voies d'exil et l'ouverture de bureaux de communication du mouvement à l'étranger, en particulier à Téhéran pour des raisons idéologiques, mais aussi à Londres. De fait, les deux composantes sociologiques de l'exil issu des monarchies du Golfe coexistent dans la capitale britannique jusqu'à nos jours, comme en témoigne la présence concomitante de princes dissidents de la famille royale saoudienne et d'exilés politiques du Bahreïn dont cet article s'efforce de retracer l'histoire des itinéraires et des itinérances, allers et retours, luttes et organisations.

\section{Les trajectoires d'exil jusqu'à l'amnistie de 2001}

Le Bahreïn connaît, historiquement, un épisode de lutte intra-élitaire conduisant au bannissement de ceux qui contestent le pouvoir en place. Au début du $X X X^{\circledR}$ siècle, l'émir du Bahreïn, Hamad bin Issa, soutenu par la puissance britannique, est confronté à une vive opposition venant de l'intérieur de la famille régnante, ainsi que de membres de tribus ou de certaines familles marchandes, traditionnellement alliés du pouvoir politique. Cette coalition de fortune s'oppose aux réformes administratives promues par les Britanniques et qui auraient donné plus de droits à la majorité de la population des villages vivant de l'agriculture et des palmeraies sur des terres affermées: elle y voit une remise en cause de sa position privilégiée. L'émir Hamad, qui avait lui-même succédé à son père, déposé par les Britanniques en 1923 en raison de sa tiédeur vis-à-vis des réformes, recourt, sous la pression ou le conseil de Britanniques, à l'expulsion des contestataires. Ainsi, les membres de la tribu sunnite des Dawassir et les fils de Khalid bin Ali (les «Khawalid»), une autre branche de la famille régnante des Khalifa, sont bannis en 1923 après que leur opposition aux réformes ait pris un tour violent et qu'ils aient procédé à des attaques contre des villageois des palmeraies. Ces derniers constituent ce que la sociologie bahreïnienne appelle les «Baharna» ou, en d'autres termes, les habitants arabes et chiites qui résidaient au Bahreïn lors de sa conquête, en 1783, par la dynastie régnante des Khalifa et ses alliés tribaux. En flagrante contradiction avec la volonté britannique de mettre sur pied un système judiciaire en bonne et due forme, les Khawalid sont condamnés à deux reprises à l'exil en Inde - peine à laquelle ils parviennent à échapper - tandis que les Dawassir s'exilent vers Dammam, sur la côte de l'actuelle Arabie saoudite, et voient leurs propriétés confisquées jusqu'en 1927, lorsque Hamad bin Issa revient sur ces décisions.

Par la suite, une nouvelle forme d'opposition politique apparaît, qui s'appuie sur une base populaire et s'inspire des mouvements anti-impérialistes, qu'ils soient nationalistes arabes ou de gauche, socialistes ou communistes. Ces mouvements populaires se heurtent à une répression violente de l'appareil étatique (avant même l'indépendance), ce qui pousse de nombreux Bahreïniens à fuir leur pays. Dans les années 1960 et 1970, la première vague d'exil politique se compose de militants révolutionnaires, anticoloniaux aux idées socialistes, marxistes ou panarabes issus de l'intelligentsia urbaine de Mamana. Elle quitte le Bahreïn à la suite de la répression qui s'abat sur la grande grève de 1965 et, une décennie plus tard, à la suite de celle qui clôt la première tentative d'expérience parlementaire du pays, entre 1973 et 1975. 
Ces premiers exilés, souvent volontaires, mais auquel l'accès au territoire bahreïnien est interdit, trouvent alors refuge en République populaire démocratique du Yémen (ou Yémen du Sud), du fait de leurs affinités idéologiques avec ce régime communiste. Certains avaient en effet soutenu ou participé au mouvement de libération du Dhofar voisin, en Oman. Ils rejoindront par la suite la Syrie bassiste après un détour par le Liban bientôt en proie à la guerre civile. Damas constitue alors un pôle d'attraction pour les oppositions de gauche du monde arabe, la République arabe syrienne délivrant sans condition des visas de résidence aux nationaux de l'ensemble des pays arabes. L'universitaire Abdulhadi Khalaf, élu député en 1973, se distingue, au sein de ce groupe, en quittant son exil libanais pour rejoindre la ville de Lund, en Suède, en 1990, rejoignant alors une autre tradition d'exil politique bahreïnien vers des pays occidentaux.

Cette seconde tradition d'exil se compose des militants et des leaders de l'opposition inspirée de l'islam politique chiite. C'est elle qui la première va trouver la route vers Londres, dans les années 1980. Ces militants appartiennent principalement à deux branches de l'islam politique chiite qui trouvent leur origine, en Irak, dans les désaccords sur les stratégies 
à suivre face à la sécularisation de la société et à l'autoritarisme du pouvoir. Le mouvement shirazi, partisan d'une stratégie de confrontation avec le régime (et plus tard enthousiaste de la révolution iranienne), se développe à Kerbala en réaction à la ligne du mouvement de la Da'wa, qui le précède historiquement et émane de l'école de pensée de Najaf. Cette dernière voit dans l'islam politique une façon de contrer l'influence grandissante des mouvements de gauche athéistes au sein de la société irakienne. Les mouvements bahreïniens reflètent cette opposition. Créée en 1972, la branche bahreïnienne de la Da'wa fournit la plupart des députés, regroupés au sein du «bloc religieux» dans l'éphémère parlement de 1973-1975, y compris le Cheikh Issa Qassem, chef spirituel de la communauté chiite bahreïnienne. Dans le contexte de la révolution iranienne et sur fond de troubles suscités par l'exécution en Irak du religieux chiite de Najaf, Mohammed Baqer Al-Sadr en 1980, une tentative supposée de coup d'État en décembre 1981, attribuée aux shirazis bahreïniens, déclenche

18 C'est aussi à Londres que fut fondée, en 1990, une dernière organisation importante de l'opposition en exil, le Bahrain Freedom Movement (BFM, Harakat al-Ahrar al-Bahrayn al-Islamiyya) à l'initiative de Saïd Al-Chehabi, Majid Al-Alaoui et Mansour Al-Jamri.

une vague de répression, de déportations et d'exils. Tandis que certains militants sont déportés vers l'Iran, d'autres prennent le chemin de pays occidentaux réputés accueillants en matière de reconnaissance de l'asile politique, à savoir le Canada, le Danemark, la Suède, mais aussi le Royaume-Uni. Comme Damas une décennie plus tôt pour les nationalistes arabes, Londres constitue à son tour un pôle d'attraction pour les exilés politiques du monde arabe issus de mouvements d'opposition islamiste, aussi bien chiites - comme dans le cas du Bahreïn ou de l'Irak - que sunnites, à l'instar des quelques membres du mouvement islamiste tunisien Ennahda. Dans le cas des pays du Golfe, anciens territoires sous protection britannique, ce rôle d'accueil des demandeurs d'asile se conjugue avec celui de destination privilégiée pour la poursuite des études universitaires.

\section{Une politique d'accueil des exilés islamistes}

Parmi les figures de cette nouvelle vague d'exil, la personnalité de Saïd Al-Chehabi mérite d'être plus particulièrement mentionnée: diplômé en ingénierie de la City University de Londres, il devient un membre de la direction de la Da'wa en Europe en 1978, position qu'il conserve jusqu'en 1985. Lorsque les Khalifa prirent connaissance, vers 1983, des activités de la Da'wa, il lui fut conseillé de ne pas rentrer au Bahreïn. Londres devient alors la base arrière de la branche bahreïnienne de la Da'wa, ainsi qu'un lieu d'exil et de rassemblement pour de nombreux exilés politiques arabes et islamistes chiites. De 1983 à 1999, Saïd $\mathrm{Al}$-Chehabi mène alors une carrière de journaliste en éditant depuis Londres l'hebdomadaire Al-'Alam («Le drapeau»), avant d'y fonder, en 1986, l'Abrar Islamic Foundation, une institution consacrée à l'étude et à la diffusion de la pensée musulmane chiite duodécimaine et aux besoins éducatifs des musulmans du Royaume-Uni. La même année, également à Londres, un petit groupe de Baharna émigrés établit Dar Al Hikma - littéralement «Maison de la sagesse »-dans l'objectif d'organiser des événements religieux, culturels, intellectuels et politiques. Les deux institutions jouent le rôle de plateforme de rencontre entre les exilés des différents pays du golfe, mettant ainsi ces derniers en contact avec le reste de l'opposition islamiste basée à Londres: parmi les soutiens de Dar Al Hikma figurent des clercs chiites venant d'Irak et d'Iran, de même que des exilés islamistes sunnites comme le Tunisien Rachid Ghannouchi ou le Frère musulman égyptien Kamel Al-Hilbaoui. Ainsi, Innes Bowen, auteur de l'ouvrage, Medina in Birmingham, Najaf in Brent: Inside British Islam', déclarait-elle lorsqu'elle en fit la présentation à Abrar House: «ll y a des liens entre des gens comme Saïd Chehabi et certains membres des Frères musulmans arabes venus ici (à Londres) depuis les années 1960 et qui ont mis en place des structures comme la Fédération des sociétés islamiques et certaines des fraternités qui furent formées alors et qui sont toujours fortes aujourd'hui².»

\footnotetext{
1. Innes Bowen, Medina in Birmingham, Najaf in Brent: Inside British Islam, Londres, Hurst, 2014.

2. Voir Abraronline.net. Url: http://www.abraronline.net/en/index. php?option=com_content\&view=article\&id=18411:islam-inbritain-who-runs-the-mosques\&catid=58:abrarmeetings\&ltemid=291. Traduction de l'auteur.
} 
C'est aussi à Londres que fut fondée, en 1990, une dernière organisation importante de l'opposition en exil, le Bahrain Freedom Movement (BFM, Harakat al-Ahrar al-Bahrayn al-Islamiyya) à l'initiative de Saïd Al-Chehabi, Majid Al-Alaoui et Mansour Al-Jamri. Ce dernier, fils d'Abdelamir Al-Jamri, un clerc chiite du Bahreïn très respecté, éduqué à Najaf et élu membre du parlement en 1973, a aussi fait ses études au Royaume-Uni (en Écosse) avant de choisir de retourner vivre à Londres en 1987. Le BFM, un mouvement proprement politique cette fois, a joué un important rôle de courroie de transmission d'informations lors du soulèvement des jeunes Bahreïniens dans les années 1990, dont Abdelamir Al-Jamri était une figure de proue. En outre, lorsque les leaders du soulèvement de 1994 au Bahreïn, en particulier le jeune cheikh Ali Salman, sont expulsés en janvier 1995, ils décident de rejoindre le BFM à Londres alors qu'ils sont en transit à Dubaï. Au Royaume-Uni, connu pour sa politique d'accueil d'islamistes persécutés dans leurs pays d'origine, la petite communauté d'exilés bahreïniens s'intègre au sein de la plus large communauté d'exilés chiites, en particulier d'origine irakienne. Mais elle interagit aussi avec les autres militants islamistes exilés à Londres, cette fois-ci sunnites, en particulier des Tunisiens, des Égyptiens et des Irakiens.

Pour l'opposition bahreïnienne en exil, les années 1990 sont marquées par l'expression de revendications collectives communes à tous les divers courants politiques exilés, qu'ils soient de gauche ou davantage religieux. Les quatre principales revendications, exprimées dans les pétitions de 1992 et 1994, concernent l'élection d'un parlement, la restauration de la constitution de 1973, la levée de la loi de 1975 sur la sécurité d'État et la libération des détenus politiques, ainsi que le retour des exilés politiques. C'est aussi à cette période que les exilés politiques bahreïniens adaptent leurs discours dans un sens davantage universel, centré sur la défense des droits et libertés fondamentales, ce qui coïncide, au Royaume-Uni, avec le lancement de la «Ethical Foreign Policy» par le Parti travailliste au lendemain de son accession au pouvoir en 1997. Les deux principales institutions agissant en ce sens sont alors le Bahrain Freedom Movement et le Bahrain Center for Human Rights créé par Abdulhadi Al-Khawaja. Ancien étudiant à Londres à partir de 1977 et inspiré par le mouvement shirazi, Abdulhadi Al-Khawaja a, quant à lui, d'abord fui vers Damas avant de se diriger vers le Danemark en 1989, où il obtint l'asile politique puis, en 1992, la nationalité danoise. À Damas comme à Copenhague, il s'investit dans la création d'organisations dénonçant les détentions arbitraires, la torture, les procès inéquitables, les dénaturalisations et les expulsions d'opposants dénaturalisés. Durant cette période de rapprochement avec les milieux politiques britanniques, le BFM professionnalise son action avec la diffusion de communiqués de presse, l'organisation de manifestations, de démarches auprès de membres du parlement de Westminster, de plaidoyer en faveur des droits de l'homme. Une des meilleures illustrations en est la création du site Internet Voice of Bahrain, bilingue arabe-anglais, consacré à l'information, sans ménagement, sur les violations des droits de l'homme dans l'archipel.

\section{Le retour des exilés et le soulèvement de 2011}

L'année 2001 marque une date importante de l'histoire des exilés du Bahreïn. Deux ans après son arrivée au pouvoir, le souverain Hamad accorde une amnistie politique générale, autorisant ainsi le retour des exilés politiques. Cette décision s'inscrit dans le cadre d'une volonté de rupture avec le règne de son père le Cheikh Issa, et avec la précédente décennie marquée par la protestation et la violence, décennie que les Bahreïniens mobilisés appellent la décennie d'Intifada. Le nouveau roi autoproclamé entend entamer un processus de changement politique décrit par certains analystes comme une "réforme institutionnelle sans partage du pouvoir ${ }^{3} »$. Amnistiés, la plupart des opposants exilés optent pour un retour vers le Bahreïn - à l'exception notable de Saïd Al-Chehabi, qui refuse tout compromis avec le régime en place.

En conséquence, au début des années 2000, l'influence du BFM diminue en raison du retour au Bahreïn de tous ses membres. Après des décennies d'activisme affiché à Londres, d'anciens exilés négocient leur retour avec un régime disposé à les réintégrer ou à les coopter. Ainsi, Majid Al-Alaoui rentre au Bahreïn pour y être nommé ministre du Travail après avoir accordé son soutien à la campagne de 2000-2001 en faveur de la charte d'action nationale promue par le nouveau roi, Hamad bin Issa. Quant à Mansour Al-Jamri, il est

3. Marina Ottaway, Michele Dunne, « Incumbent Regimes and the "King's Dilemma" in the Arab World: Promise and Threat of Managed Reform », Carnegie Papers, n 88, 2007, Carnegie Endowment for International Peace, Middle East Program, p. 6. 
autorisé en 2002 à créer au Bahreïn le premier et seul journal indépendant d'opposition à ce jour, Al-Wassat, fermé définitivement en 2017.

Avec cette timide ouverture de la vie politique bahreïnienne, les anciens exilés politiques de Londres peuvent, à partir de 2002, établir au Bahreïn des «associations politiques», qui sont le reflet des différents mouvements d'opposition existant depuis l'indépendance. Dirigée par le cheikh Ali Salman, l'association nationale islamique Al-Wifaq («la concorde»), en particulier, est créée comme un mouvement visant à rassembler l'ensemble des courants idéologiques chiites, à l'exception du courant shirazi. Subrepticement, la libéralisation de la vie politique locale, bien que prudente, l'emporte alors sur la vie politique en exil. Tout

\section{(1. Assoupis par la timide ouverture politique des années 2000, les réseaux d'opposition bahreïniens de Londres voient arriver nombre de ces nouveaux exilés, dont d'anciens députés du Wifaq, et se remobilisent alors.}

au long des années 2000, le BFM conserve sa ligne politique de non-compromis avec la monarchie et Londres demeure le point focal de l'opposition bahreïnienne en exil. De nombreux opposants peuvent circuler librement entre la capitale britannique et l'archipel, tandis que le BFM est rejoint dans sa ligne politique, mais aussi en exil, par des dissidents du Wifaq, en désaccord avec sa décision de participer aux élections législatives de 2006 après quatre ans de boycott. Il est également rejoint par les membres de deux nouveaux mouvements Al-Haqq et $A l-W a f a$ ', qui refusent d'accorder la moindre confiance à la dynastie des Khalifa. Le BFM, Al-Haqq et Al-Wafa'se font les avocats du boycott des élections parlementaires de 2010 - ce sont eux qui appelleront, le 8 mars 2011, au «renversement du régime par des manifestations pacifiques et [à] l'établissement d'une république démocratique». Illustration de cette tension renaissante, à l'été 2010, l'opposant et membre d'Al-Haqq, Abduljalil Al-Singace, se rend à Londres pour y témoigner devant la Chambre des lords britanniques. Mais, à son retour au Bahreïn le 13 août 2010, il est arrêté et torturé.

Lorsque survient le soulèvement de 2011, Hassan Al-Mushaïma, l'un des leaders du mouvement Al-Haqq, alors en traitement médical à Londres, s'adresse depuis la capitale britannique à la foule des manifestants rassemblés sur le rond-point de la Perle, allocution retransmise via Internet sur des écrans géants, avant de retourner au Bahreïn le 26 février 2011.

Après une décennie de répit, la répression brutale des protestations populaires de 2011 marque le début d'une nouvelle vague d'expulsions et de départs forcés, d'une ampleur sans précédent dans la mesure où elle touche aussi des personnes dont le rôle politique est périphérique. C'est le cas de journalistes ou d'employés licenciés car soupçonnés de sympathies pour l'opposition, notamment du fait de leur absence de leur lieu de travail au moment de manifestations. Le départ en exil de journalistes bahreïniens favorise également l'éclosion de nouveaux médias, tels que le site internet Bahrain Mirror (basé au Liban) ou bien encore la chaîne de télévision Lulua TV, proche de la ligne politique du Wifaq et diffusant depuis une banlieue londonienne. Assoupis par la timide ouverture politique des années 2000, les réseaux d'opposition bahreïniens de Londres voient arriver nombre de ces nouveaux exilés, dont d'anciens députés du Wifaq, et se remobilisent alors. Parmi ces derniers, nombreux sont ceux qui se verront déchus de leur nationalité et se retrouveront en situation d'apatridie au Royaume-Uni.

\section{L'émergence d'une nouvelle génération d'exilés politiques}

L'opposition en exil voit de nouvelles formes d'organisations, promues par une nouvelle génération d'activistes, telle Bahrain Watch, une structure créée en 2012 par une dizaine de jeunes, exilés bahreïniens ou activistes ayant vécu dans le royaume, pour la plupart diplômés d'universités occidentales prestigieuses, parmi lesquels Ala'a Al-Chehabi, la fille de Saïd Al-Chehabi. Née à Londres, cette dernière y a grandi et étudié; elle a obtenu son doctorat d'économie au Royaume-Uni avant de s'installer au Bahreïn en 2009 et de devenir, dès les débuts du soulèvement de 2011, une figure publique de l'opposition bahreïnienne à la suite de l'arrestation de son mari. Bahrain Watch incarne, depuis Londres, une rupture avec les idéologies religieuses, islamistes et gauchistes des précédents mouvements d'opposition bahreïniens en regroupant des activistes de nationalités diverses et de statuts différents lors de leurs séjours au Bahreïn (enfants d'expatriés, deuxième génération de migrants).

Outre Ala'a Al-Chehabi, les années 2010 sont marquées par l'émergence d'autres figures de la 
deuxième génération de militants politiques bahreïniens, au Bahreïn comme en exil, tels Ali Al-Mushaïma, fils de Hassan Al-Mushaïma, le leader d'Al-Haqq, condamné à une peine de prison, et les sœurs Maryam et Zeynab Al-Khawaja, filles d'Abdulhadi Al-Khawaja. Maîtrisant l'intégralité des codes éducatifs, culturels, juridiques, de lobbying et de communication politique des pays occidentaux, cette nouvelle génération de militants politiques bahreïniens en possède généralement aussi les nationalités (britannique, danoise, suédoise). Si cette plurinationalité lui offre une forme de protection politique (mais non juridique), elle lui vaut aussi d'être la cible de mesures spécifiques de la part du gouvernement bahreïnien. Ce dernier, oscillant entre accusations de déloyauté et dénaturalisations, annonce en avril 2012 un durcissement des mesures prises contre ceux qui n'ont pas notifié une deuxième nationalité et régularisé leur statut auprès du ministère de l'Intérieur au titre de l'article 9 de la Loi sur la citoyenneté de 1963.

Du fait de son positionnement, cette nouvelle génération d'exilés politiques parvient à brouiller les hiérarchies sociales bahreïniennes, mais aussi à élargir le cercle des soutiens de l'opposition bahreïnienne à des sympathisants et des militants de pays occidentaux ou de pays arabes comme l'Égypte ou la Tunisie. Elle n'hésite pas non plus à poursuivre en justice des membres de la famille régnante bahreïnienne jusqu'ici considérés comme intouchables au Royaume-Uni. Ainsi, en octobre 2014, un réfugié politique bahreïnien parvient à obtenir de la justice britannique une décision indiquant que le fils du roi du Bahreïn, Nasser bin Hamad Al-Khalifa, éduqué à Sandhurst et voyageant fréquemment au Royaume-Uni, ne bénéficie pas d'une immunité juridictionnelle après qu'il ait été accusé d'avoir recouru à la torture.

Ces évolutions traduisent aussi une forme d'«effet boomerang» pour le Royaume-Uni qui, de pays d'accueil d'opposants bahreïniens, se retrouve aussi en position d'accusé en raison du soutien constant et de l'assistance de tous ordres - notamment policière - qu'il a apporté à la dynastie des Al-Khalifa tout au long de son histoire, et continue de lui apporter. 